\title{
Secure Data Transfer Guidance for Industrial Control and SCADA Systems
}

RE Mahan

JD Fluckiger

SL Clements

C Tews
JR Burnette

CA Goranson

H Kirkham

September 2011

Pacific Northwest

NATIONAL LABORATORY

Proudly Operated by Battelle Since 1965 


\title{
DISCLAIMER
}

This report was prepared as an account of work sponsored by an agency of the United States Government. Neither the United States Government nor any agency thereof, nor Battelle Memorial Institute, nor any of their employees, makes any warranty, express or implied, or assumes any legal liability or responsibility for the accuracy, completeness, or usefulness of any information, apparatus, product, or process disclosed, or represents that its use would not infringe privately owned rights. Reference herein to any specific commercial product, process, or service by trade name, trademark, manufacturer, or otherwise does not necessarily constitute or imply its endorsement, recommendation, or favoring by the United States Government or any agency thereof, or Battelle Memorial Institute. The views and opinions of authors expressed herein do not necessarily state or reflect those of the United States Government or any agency thereof.

\author{
PACIFIC NORTHWEST NATIONAL LABORATORY \\ operated by \\ BATTELLE \\ for the \\ UNITED STATES DEPARTMENT OF ENERGY \\ under Contract DE-AC05-76RL01830 \\ Printed in the United States of America \\ Available to DOE and DOE contractors from the \\ Office of Scientific and Technical Information, \\ P.O. Box 62, Oak Ridge, TN 37831-0062; \\ ph: (865) 576-8401 \\ fax: (865) 576-5728 \\ email: reports@adonis.osti.gov \\ Available to the public from the National Technical Information Service,
U.S. Department of Commerce, 5285 Port Royal Rd., Springfield, VA 22161 \\ ph: (800) 553-6847 \\ fax: (703) 605-6900 \\ email: orders@ntis.fedworld.gov \\ online ordering: http://www.ntis.gov/ordering.htm
}




\section{Secure Data Transfer Guidance for Industrial Control and SCADA Systems}

RE Mahan

JD Fluckiger

SL Clements

C Tews
JR Burnette

CA Goranson

H Kirkham

September 2011

Prepared for the U.S. Department of Energy

under Contract DE-AC05-76RL01830 
PNNL-20776

Pacific Northwest National Laboratory Richland, Washington 99352 


\section{Table of Contents}

Secure Data Transfer Guidance for Industrial Control and SCADA Systems ............................ 1

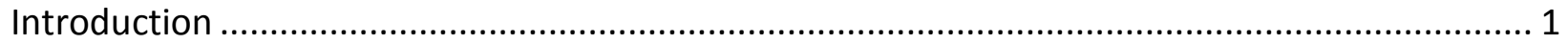

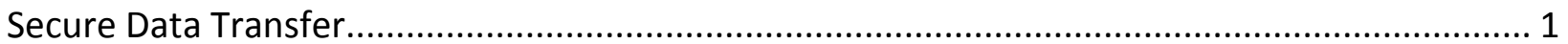

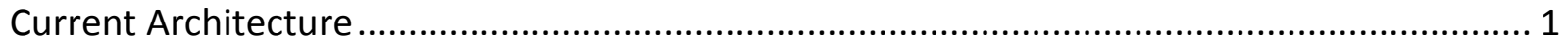

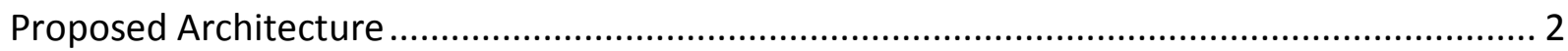

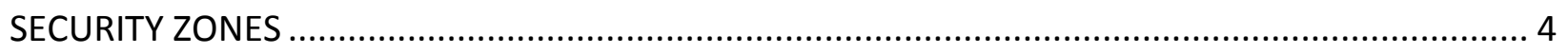

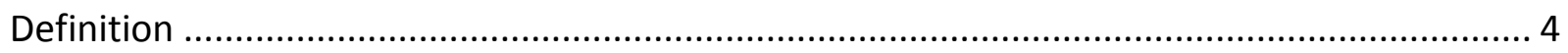

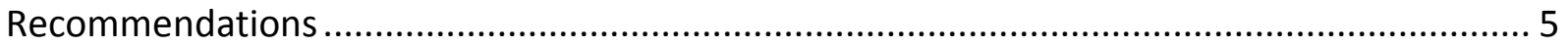

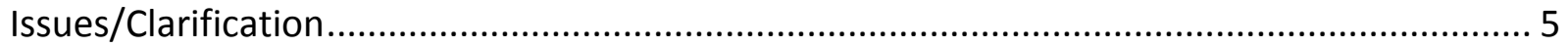

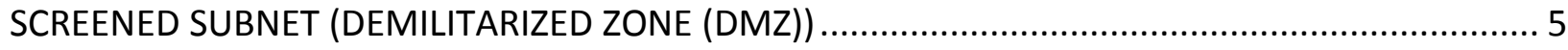

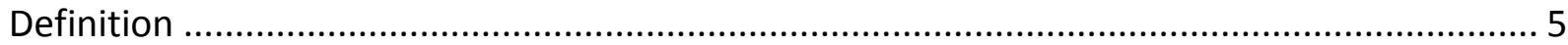

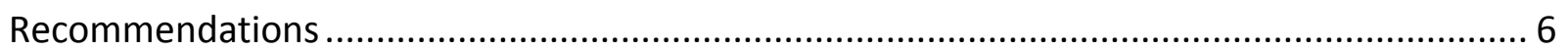

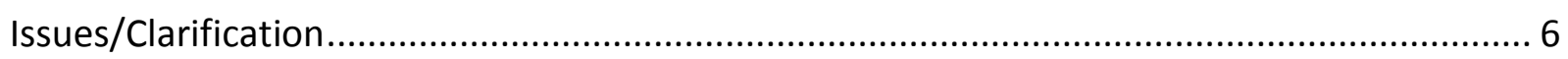

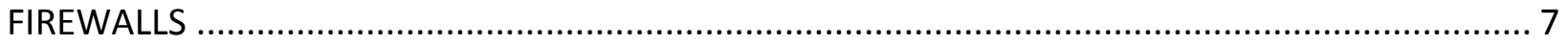

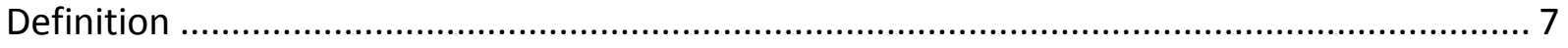

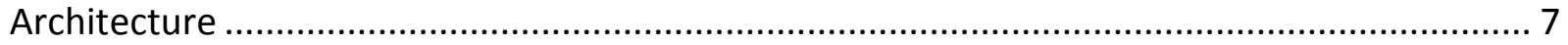

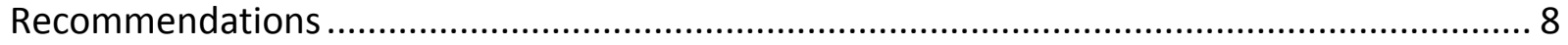

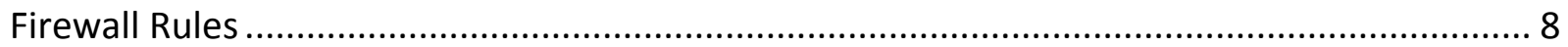

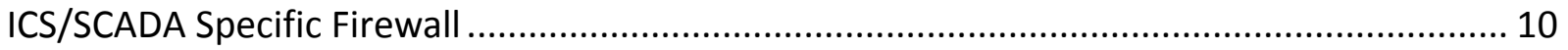

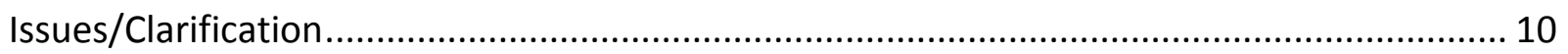

INTRUSION DETECTION SYSTEM/INTRUSION PROTECTION SYSTEM (IDS/IPS) ........................ 11

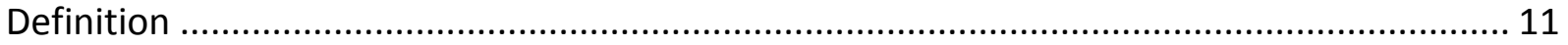

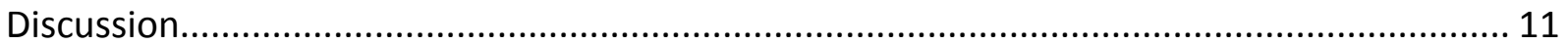

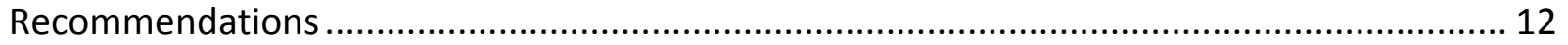

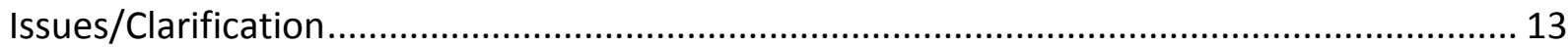

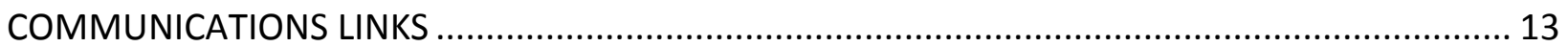

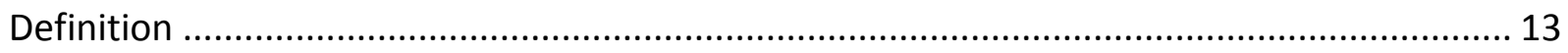

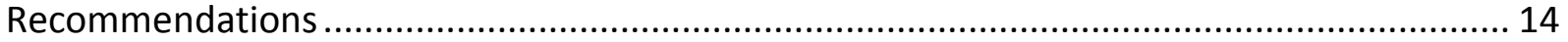

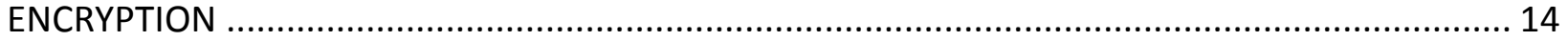

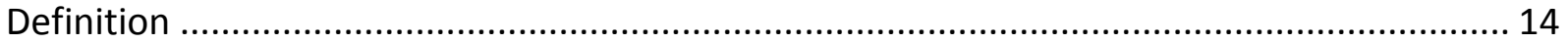

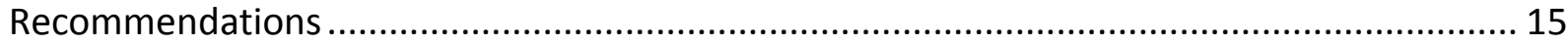




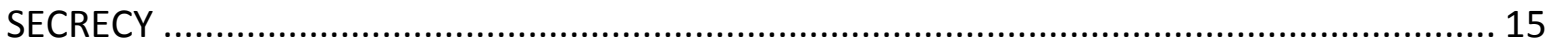

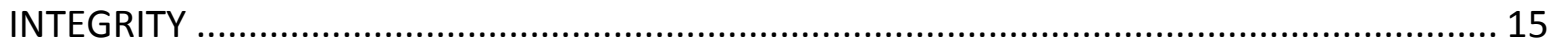

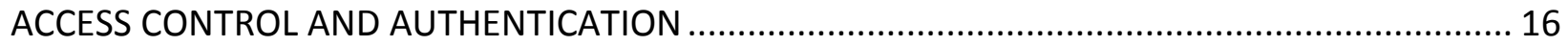

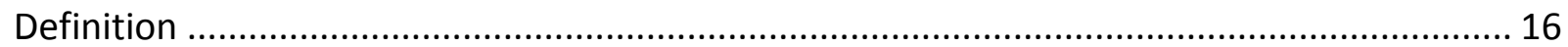

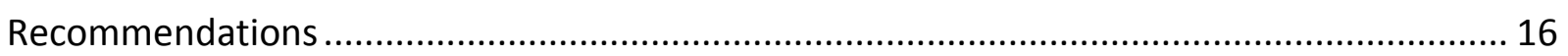

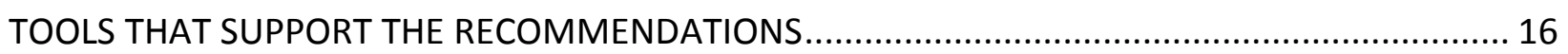

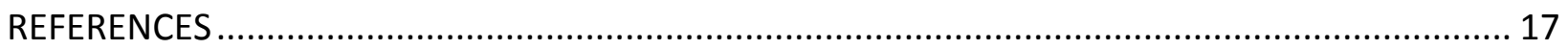

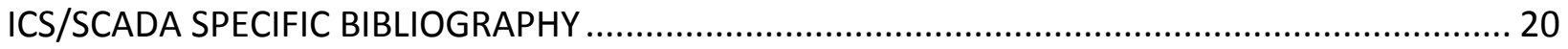

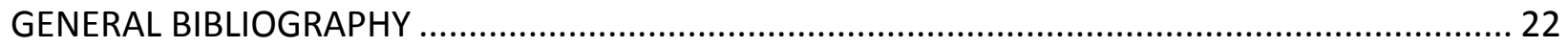

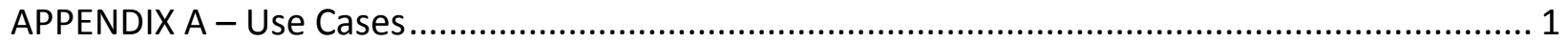

Use Case 1: Enterprise Network to Operations Network.................................................... 1

Use Case 2: Operations Network to External Control Center Network (Phasor Data). .............. 2

Use Case 3: Operations Network to External Control Center Network - ICCP Data................... 3

Use case 4: Operations Network to NERC, Regulatory Data..................................................... 4

Use Case 5: Control Center Network to Vendor Network, Support \& Maintenance.................. 5

Use Case 6: Operations Network to Automated Meter Reading. ............................................. 6 


\section{Introduction}

This document was developed to provide guidance for the implementation of secure data transfer in a complex computational infrastructure representative of the electric power and oil and natural gas enterprises and the control systems they implement.

For the past 20 years the cyber security community has focused on preventative measures intended to keep systems secure by providing a hard outer shell that is difficult to penetrate. Over time, the hard exterior, soft interior focus changed to focus on defense-in-depth adding multiple layers of protection, introducing intrusion detection systems, more effective incident response and cleanup, and many other security measures. Despite much larger expenditures and more layers of defense, successful attacks have only increased in number and severity.

Consequently, it is time to re-focus the conventional approach to cyber security. While it is still important to implement measures to keep intruders out, a new protection paradigm is warranted that is aimed at discovering attempted or real compromises as early as possible.

Put simply, organizations should take as fact that they have been, are now, or will be compromised. These compromises may be intended to steal information for financial gain as in the theft of intellectual property or credentials that lead to the theft of financial resources, or to lie silent until instructed to cause physical or electronic damage and/or denial of services. This change in outlook has been recently confirmed by the National Security Agency [19].

The discovery of attempted and actual compromises requires an increased focus on monitoring events by manual and/or automated log monitoring, detecting unauthorized changes to a system's hardware and/or software, detecting intrusions, and/or discovering the exfiltration of sensitive information and/or attempts to send inappropriate commands to ICS/SCADA (Industrial Control System/Supervisory Control And Data Acquisition) systems.

\section{Secure Data Transfer}

\section{Current Architecture}

A generic Industrial Control System/SCADA architecture was documented as shown in Figure 1 to indicate the general functionality typical of existing systems (although actual implementations are highly variable). In addition, 6 use cases are documented at the interfaces shown by the red dots (1-6) in Figure 2 to indicate locations requiring protection. These use cases are shown in greater detail in Appendix 1. 
Figure 1. Generic ICS/SCADA Architecture

\section{Generic Control System Architecture}

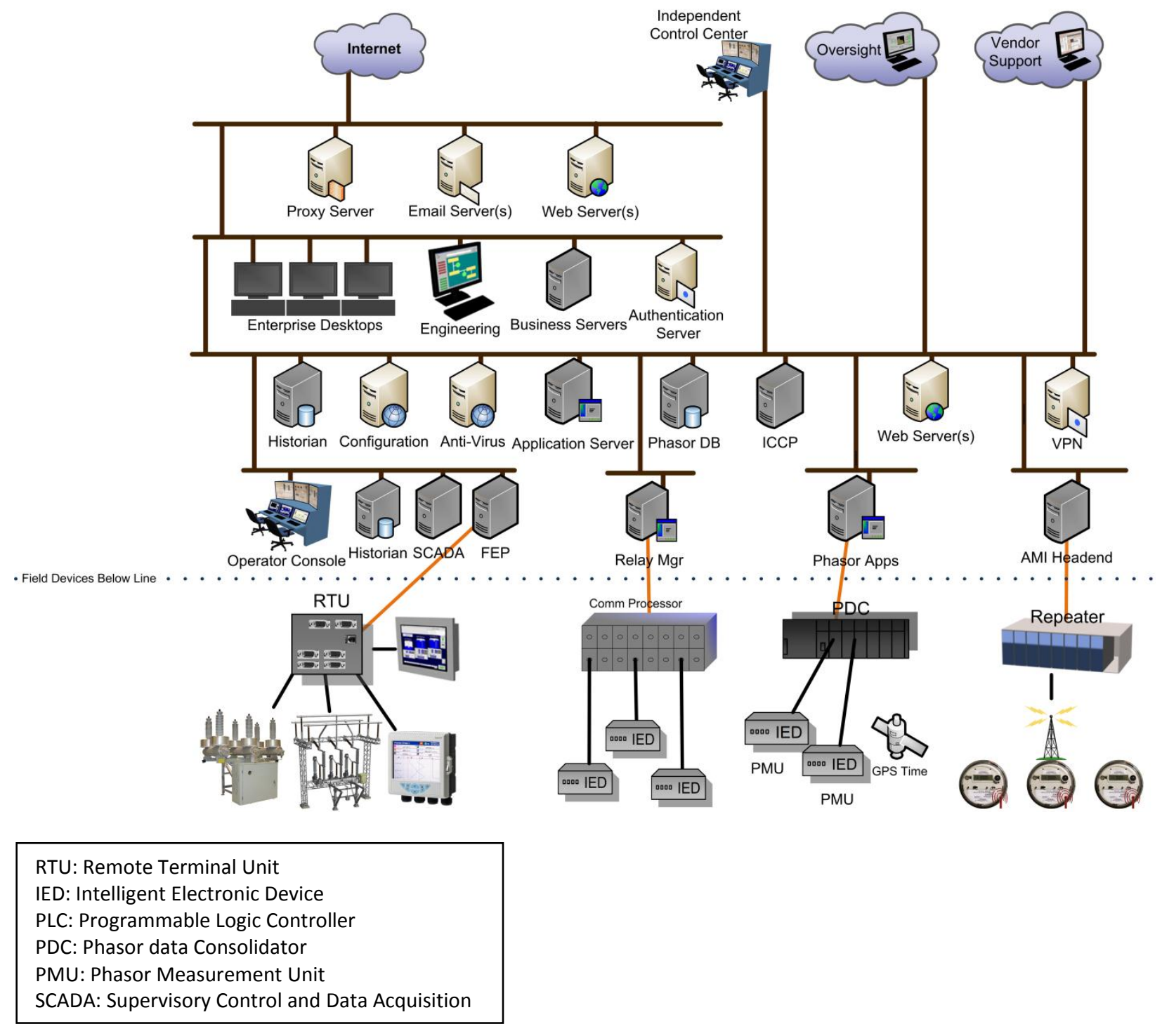

\section{Proposed Architecture}

The proposed architecture shown in Figure 2 presents the arrangement of Figure 1 with the addition of the security recommendations made in this document including: 1) improved security for the corporate/enterprise network connection to the Internet; 2) a recommended security zone architecture intended to isolate critical networks; and 3) the addition of multiple screened subnets/Demilitarized Zones (DMZ) with no transit traffic.

It is important 1) that the implementation of the proposed architecture should allow no connections between security zones that are not firewall protected; 2 ) that an inventory of all remote access paths that enter the Generic architecture be completed to ensure there are no 
connections that bypass the firewall infrastructure; and 3) that remote access be through a VPN connection with strong access controls (i.e., at least 2 factor authentication).

Figure 2. Proposed SCADA Architecture

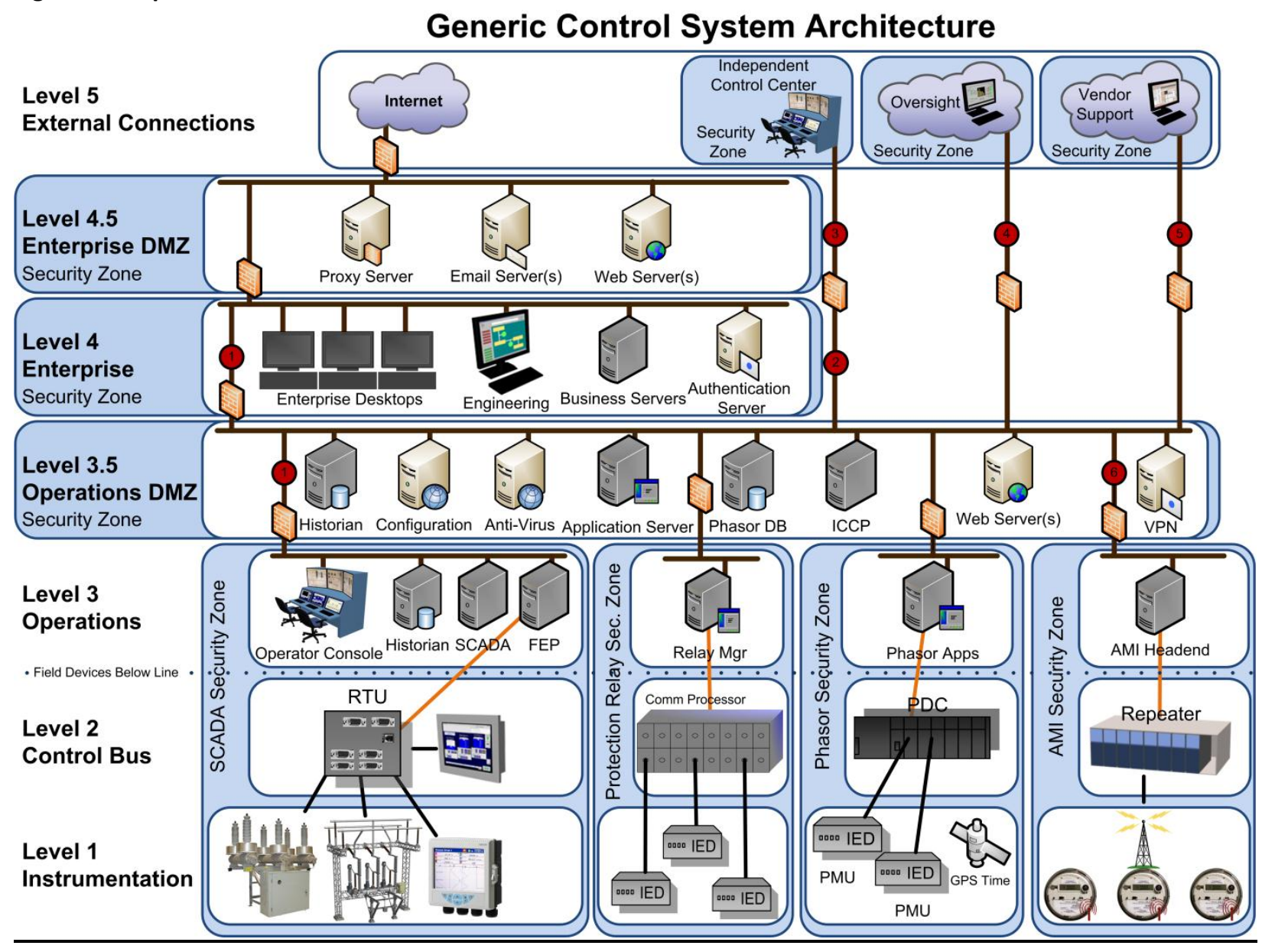

The red circles on the diagrams indicate use cases that were considered while authoring this guide. Six specific use cases were considered and are presented in greater detail in Appendix A. Use case 1 is the connection between the Enterprise network and the Operations network. Use case 2 is the connection between control centers that share phasor data. Use case 3 is the ICCP connection between control centers. Use case 4 is the connection that supports regulatory reporting (e.g., to NERC). Use case 5 is any connection established to support vendor activities (e.g., monitoring and maintenance). Use case 6 is the connection that supports data collection from smart meters. Each one of these connections needs to be protected since each of these paths represents a potential channel for compromising one, or more, systems that reside in the architecture. Not shown is a general enterprise wireless network. If one is present it must be protected from intrusion and compromise.

The Engineering workstations might logically reside inside the enterprise network, in its own dedicated security zone, or as a part of the operations network. In any case, since engineering 
often has a requirement for direct access to control center systems or systems at remotes sites (e.g., generation, distribution, etc.) these systems must be well protected.

The remainder of this document describes the recommended implementation of the proposed architecture.

\section{SECURITY ZONES}

\section{Definition}

A security zone is a collection of information systems connected by one, or more, internal networks under the control of a single authority and one security policy. The systems may be structured by physical proximity or by function, and may be independent of location [1]. For example, engineering and business operations may be located in the same building or distant facilities, but share common functions like e-mail and general web services. Consequently, it is often decided to locate them in the enterprise security zone.

Control centers perform significantly different functions, have some common requirements (e.g., e-mail), and support active communications and control with local and/or remote sites. The need for common services delivered to all locations across the entire enterprise argues for a single enterprise network, while the need to protect critical resources argues against a common network. The most effective solution to this situation is to segregate the enterprise network into security zones such that necessary services can be supported, but critical resources can be protected.

The most important characteristic of a security zone-based architecture is that all systems residing within a specific zone are protected under the same policy (i.e., all systems must implement at least the minimum level of protection specified for the zone). Conversely, it is acceptable for the protection of individual systems within a zone to exceed minimum specified requirements depending on the criticality of the system.

A security zone must have a well-specified boundary, and communication between zones must be filtered in accordance with policy. Services, protocols, and applications that are not essential within a zone should be disabled within the zone. Any boundary-crossing traffic carrying those services, protocols, or applications should be blocked at the boundary. For example, ICS/SCADA protocols such as Modbus TCP or DNP3 are required components for controlling process devices. Whenever possible, these protocols should be disallowed outside the Operations security zone. If that is not possible, then compensating measures need to be taken to protect them from attack and/or misuse from sources outside the process control network. 


\section{Recommendations}

A zone-based architecture is recommended to strengthen the capability to perform secure data transfers across the generic SCADA architecture.

Segment the architecture into at least 5 security zones (External Connections, Enterprise DMZ, Enterprise, Operations DMZ, and Operations).

\section{Issues/Clarification}

In some of the literature, security zones are referred to as enclaves. This term is most prevalent in IT security practices.

Our model loosely follows the zone model described in the Purdue Reference Model (PRM) for Computer Integrated Manufacturing of IEC Standard 62254-1 and reference model IEC Standard 622443-1 that suggests a model with 6 zones [2].

Zone 1: Automation: Process (equipment under control), Safety \& protection, Basic control/Local control),

Zone 2: Operation Control: Supervisory control,

Zone 3: Operation support: Operation management,

Zone 4: Business support: Business planning and logistics,

Zone 5: Corporate IT: Enterprise IT \& common services, and

Zone 6: External Integration: Connections and information transfer to third parties.

ANSI/ISA exhibits a 4 zone model [3].

Enterprise Zone: Corporate systems

Control Center Zone: Primary and Backup Control Centers.

Demilitarized Zone: Historian and Remote Operator Console.

Site Control Zone: Local Operator and Engineering workstations, servers, and ICS/DCS/SCADA devices.

\section{SCREENED SUBNET (DEMILITARIZED ZONE (DMZ))}

\section{Definition}

A screened subnet (or DMZ) is a perimeter network segment that is logically between two networks. Its purpose is to enforce the internal network's Information Assurance (IA) policy for external information exchange and to provide external, untrusted, sources with restricted access to information that needs to be released outside the protected network while shielding the internal network from outside attack [4].

The most common DMZ architecture uses a firewall with three network interfaces between two networks. Interface 1 connects to the external network (e.g., the Internet). Interface 2 
connects to the protected DMZ (e.g., the public web servers hosted by the organization) and interface 3 connects to an internal network (e.g., the enterprise network).

\section{Recommendations}

Implement an external DMZ to provide public access to external (e.g., Internet) facing servers (e.g., enterprise web services) as provided by corporate policy. No transit traffic is allowed across servers located in the DMZ. Data is pushed to the servers located in the DMZ from the enterprise network and accessed by the public.

Add an internal DMZ between the enterprise network and the operations network(s). An Internal DMZ provides enterprise access to operations information, typically the Historian that holds data from the ICS/SCADA network and remote stations. No transit traffic is allowed across servers located in the internal DMZ. As a corollary, the historian should not be located on the enterprise network or the ICS/SCADA network but should reside in the DMZ, though some secure implementations utilize mirrored historians in the operational network and in the internal DMZ. Similarly, any server (e.g., a data server or patch management server) that is jointly accessed from the enterprise network and the ICS/SCADA network should be located in the $\mathrm{DMZ}$ and transit traffic blocked. In addition, special attention should be paid to locking down DMZ servers (e.g., removing all unnecessary services, whitelisting IP/MAC addresses to limit access to only those workstations approved by policy) In addition, traffic should be subjected to port filtering disallowing unnecessary services between security zones. The only devices that should exist in the $\mathrm{DMZ}$ are devices that store data that needs to be shared between networks (e.g., Historian).

Multiple segregated DMZs at either Level 3.5 or 4.5 may be necessary if the architecture requires. In Figure 2 it is likely that the ICCP server would be in its own DMZ separate from the other systems at that same level in the hierarchical model.

\section{Issues/Clarification}

In most of the literature, a DMZ means a protected network where information is available to the public. In this discussion, that network is called the external DMZ. The internal DMZ is not accessible to the public, but is accessible from the enterprise network. It is intended to protect the SCADA control center and remotes sites from the enterprise network and any entity that is provided SCADA information (e.g., regulatory bodies like NERC). Thus, a compromise of a system in the enterprise or regulatory network will not compromise the SCADA network. 


\section{FIREWALLS}

\section{Definition}

A firewall is a hardware/software capability that limits access between networks and/or systems in accordance with a specific security policy [5].

\section{Architecture}

There are four general firewall architectures: packet filtering, stateful inspection, application layer, and bastion hosts (the latter are also variously called multi-homed hosts, gateways, or proxies).

Packet filters operate at the network layer (layer 3) of the Transmission Control Protocol (TCP) model. Pure packet filtering firewalls check the packet header (typically for type, and source and destination address) but do not examine the information in the transport layer (layer4) or application layer (layer 5). Consequently, attacks on layers $4 \& 5$ are typically neither blocked nor detected. The primary advantage of packet filters are low cost and minimal impact on network performance. It is often useful to implement packet filtering in a router in front of a stateful inspection firewall. This has the dual advantage of providing defense-in-depth and reduces the workload on the stateful firewall.

Stateful inspection firewalls examine the same information as a packet filter (i.e. layer 3 ) and also adds a check on transport layer (layer 4) information. This further check allows it to handle traffic such as TCP and connectionless UDP. Such firewalls are more effective in detecting threats, but have higher cost, with greater performance impact, and are more complex to configure and operate.

One of the limitations of current stateful inspection firewalls that examine transport layer protocols is that many do not recognize ICS/DCS/SCADA protocols.

Application layer firewalls (also known as proxy servers) add the capability of examining specific application traffic including web services, FTP services, and others. Coverage for ICS/DCS/SCADA applications is presently limited and performance impact is typically greater than other firewall types. The benefits of using this method are significant, as the proxy server is the only thing significantly exposed to the untrusted network. The disadvantage of the approach is that it is application-specific. Each packet must be examined down to the application layer (which may be slow) and must be "tuned" to each application allowed.

Bastion hosts or gateways/proxies/multi-homed hosts are general purpose computers with two-network cards. For example, one might be attached to the enterprise network and the other to the ICS/SCADA network. The literature generally advises against the use of dual-homed systems because they can be more vulnerable to attack than the dual-homed firewall since they 
are hosted on a general-purpose computer. However, they are low cost solutions and have been used successfully when they have been "hardened" by:

1) turning off all but the few essential services/daemons and protocols required,

2) limiting user accounts, requiring strong authentication to access the system,

3) not sharing authentication services with trusted hosts on the network,

4) configuring to only open essential ports,

5) configuring for full logging with high integrity (read-only encrypted local logs or logged to a remote system or a read-only device),

6) shutting down as many utilities and configuration tools as is possible,

7) keeping the system fully patched,

8) removing all unnecessary applications, and

9) running an IDS on the system [13][14].

A second major differentiator of firewalls is their placement. Network firewalls are located at the ingress/egress point of a protected network (e.g., at a security zone boundary). Host-based firewalls are installed on end devices (e.g., servers, workstations). Many sites implement both network and host-based firewalls as a defense-in-depth measure.

\section{Recommendations}

Add a firewall between each security zone.

Ensure a firewall exists between the enterprise network and the SCADA network. Do not allow direct communication between the enterprise network and the ICS/SCADA network. This limitation can be implemented by the use of a Demilitarized Zone (DMZ) as described earlier in this document. If direct traffic between the enterprise zone and operations zone (e.g., engineering access) is required, provide strong authentication and encryption using, for example, a VPN connection.

Add a firewall between the wireless network and the network it connects to.

If a remote station supports a routed network, consider adding a firewall between the SCADA network and the remote station.

Perform egress filtering between zones. This will allow the blocking of malware that may have got onto the systems via mobile devices that bypass many of the security controls. This filtering also allows the detection and blocking of exfiltration traffic and unauthorized peer-to-peer traffic.

\section{Firewall Rules}

Firewall rules form the basis for filtering traffic between two networks and are designed to take one of four actions: 1) Allow/Accept the connection; 2) Allow/Accept the connection based on 
meeting a condition (e.g., encryption); 3) Block/Reject the connection with notification to the source; and 4) Block/Drop the connection without notification to the source. These actions must be specified for both in-bound and out-bound connections to/from the protected network.

Typical conditions for allowing connections require additional action such as: 1 ) authenticate the client; 2) encrypt/decrypt the payload; 3) scan for viruses; and 4) test source address against a whitelist of allowed addresses or against a black list of disallowed addresses. Whitelisting can also be implemented on servers. For example, Microsoft Server 2003 and newer can restrict applications under Group Policy allowing only certain applications to run.

Firewall rules are often created that have conflicts. Consequently the processing order of the rules is an important consideration since a preceding rule can override a following rule.

A few of the most important generic rules are as follows:

1. Block with no notification all inbound traffic destined for the firewall (Stealth rule that makes the firewall invisible to attack scanners).

2. Block all outbound traffic originated from the firewall (the firewall should not be making direct connections to external systems).

3. Block all inbound traffic where the source address is an address internal to the protected network (no inbound traffic should contain an address internal to the network).

4. Block all outbound traffic where the source address does not belong to the protected network (all outbound traffic should have a legal source address).

5. For the process control network ingress and egress traffic disallow http session, ftp session, e-Mail, telnet, ssh, DNS, and any other traffic that is not Historian master or slave traffic, specific to engineering, or vendor maintenance. If these classes of communication to the enterprise or an external network are essential to operations, they should be performed on separate devices (other than process operator consoles) and over a segregated physical or virtual network.

6. For the case of vendor maintenance, the safest path is to allow a session only during specific maintenance activities and then block it.

7. If engineering or remote maintenance access is needed from the enterprise network or any other location, it should be via a VPN tunnel that is enabled using strong authentication. Split tunnels should be disallowed.

8. $\mathrm{N}^{\text {th }}$ The last rule is the default rule and should always be Block/Deny without notification everything not explicitly allowed by the preceding rules.

This is an extremely short list of rules. Actual implementations often operate with a hundred or more rules. Consequently it is easy to establish conflicting rules and it is a good idea to run the rule set against a program such as Nipper ${ }^{\circledR}$ that analyzes rules for inconsistencies and/or conflicts [15]. There are many other tools available to aid in building, editing and auditing firewall rule sets. 
Firewalls have the capability to log traffic at multiple levels of detail. Decisions will have to be made whether to short log or long log each rule. These decisions should be made based on performance requirements and the criticality of the resource being protected.

\section{ICS/SCADA Specific Firewall}

While traditional IT firewalls used to isolate networks are generally unaware of ICS/SCADA protocols (e.g., Modbus, DNP3), there is scattered documentation available on the design and implementation of firewalls with limited ICS/SCADA awareness. In recent years many firewall vendors have begun tailoring their products to the ICS community.

Guidance for SCADA and process control firewall design is available from the UK Centre for the Protection of National Security Infrastructure at: http://www.cpni.gov.uk. There is also be information available from the Department of Homeland Security at http://www.us-

cert.gov/control systems/.

Digital Bond offers firewall rules guidance at: http://www.digitalbond.com/scadapedia/security-controls/best-practices-for-firewalls-indigital-control-and-scada-systems/

Recommendations include: 1) Authenticate before allowing configuration changes; 2) Perform Self-testing; 3) Perform Logging; and 4) Do not deploy with default settings.

A firewall is available for Modbus TCP implemented under Linux and can be found at Source Forge: A download is available at: http://modbusfw.sourceforge.net/

\section{Issues/Clarification}

Firewalls may introduce unacceptable latency in ICS/SCADA communications especially in the case of Phasor Measurement Units (PMU). Some firewall functions achieve their protection by checking packet header information (and even payload content) against lists that are maintained of acceptable and unacceptable addresses. A denial of service attack can be mounted on such a firewall by overloading it with packet to be checked. For PMU data, which are typically reported tens of times per second (much more frequently than SCADA), such attacks may be too easily able to create unacceptable delays.

The firewall may be bypassed if there are other paths that can reach ICS/SCADA devices. All entry points to the ICS/SCADA network should be configured to pass through the firewall. If that is not possible additional mitigations should be applied to the system being accessed. 
Effective firewall rules are essential to the successful implementation of protection. The rules are complex and multiple research studies have often found misconfigured firewall rule sets.

Multicast traffic is becoming increasingly popular in control environments since it reduces network load and has excellent time synchronization characteristics. However, in complex, firewall-protected networks, multicast routing becomes more complex and requires additional protocols and firewall rules.

Hopefully the information presented here illustrates that firewall configuration and maintenance is not a trivial task. Architecting and segmenting a network with security zone relies on proper firewall configurations. It is essential for a secure architecture that those responsible for firewall configuration and maintenance are properly trained and qualified.

\section{INTRUSION DETECTION SYSTEM/INTRUSION PROTECTION SYSTEM (IDS/IPS)}

\section{Definition}

Intrusion Detection System (IDS): A hardware and/or software product that gathers and analyzes information from various areas within a computer or a network to identify possible security breaches, which include both intrusions (attacks from outside the organization) and misuse (attacks or malfeasance from within organizations) [6]. Both network-based and hostbased systems are available. Host-based IDS systems monitor security within network components such as servers or workstations while a network-based IDS monitors the traffic between network components and networks.

Intrusion Prevention System (IPS): These systems are devices that can detect intrusive activity and attempt to stop the activity, ideally before it reaches its target(s) [6]. IPS systems can be recommended to detect, but automated prevention is generally not recommended at layers 3 and lower for SCADA systems, because the preventive action could result in unintended consequences.

\section{Discussion}

An early host-based method of detecting intrusions was based on the capability of a system to log security events. Early systems relied on manual methods to examine the logs on a system, or systems, for anomalous behavior. Logs tended to be large and reading them is labor intensive and often neglected because of the level of effort and monotony of the work. However, in most successful compromises, evidence of the attack can almost always be found in the logs unless the attacker has taken special care to expunge the logs of the compromising events. Advanced logging systems log the information to a read-only device or a location remote from the system being monitored, preserving the information. More recently, log 
analysis software has been developed to automatically examine logs and generate alerts from a central location. More sophisticated systems canonize the logs into a standard form, store the information in a database, and use structured queries to examine and correlate security events. OSSEC is an example of an open source host-based intrusion detection system using a centralized system for analysis and alerting [16][17]. The most important characteristic of centralized logging and analysis is the capability to automate the analysis activity and generate alerts that trigger additional manual analysis.

Another detection approach is to install a tool that calculates a cryptographic checksum (i.e., hash) on critical files, store the hashes in a secure location, and alert an administrator when an unauthorized change is made to critical files (e.g., a configuration file or registry entry is changed or a new account is added).Such a tool can also be used to monitor controlled changes. The system is examined after the authorized change to ensure that only the authorized change was made. A well-known software package that performs this function on Windows and Linux systems is Tripwire [18].

Network Intrusion Detection Systems (NIDS) record and analyze network traffic information related to security events, notify administrators of suspicious events, and generate reports. A network IDS uses one of two detection techniques; signature matching or anomaly detection. Statistical-based anomaly detection systems determine normal network activity (e.g., bandwidth used, protocols, ports, and communicating devices) and alert on anomalous traffic. Signature-based systems monitor network traffic and alert on known attack patterns. NIDS are often subject to weaknesses due to encoding that masks malicious traffic, out-of-order packet sequencing, and encrypted malware communications.

Host Intrusion Detection Systems (HIDS) perform the same functions as a NIDS using signature matching and/or anomaly detection, but are designed to overcome issues with encoding, sequencing, and encryption since they can view the incoming traffic after it is decoded, correctly sequenced, and/or decrypted. The problem is that the end device must be capable of running the HIDS and the HIDS must correctly detect the attempted intrusion. In addition, HIDS is often more expensive to implement and maintain than NIDS.

Finally, a honeypot can also be used to detect intrusions. A honeypot is a system configured to attract an intruder. It is essentially a trap set up to detect, deflect, and identify malicious acts in order to respond to and defeat attempts at compromising operational systems.

\section{Recommendations}

Implement remote syslog for auditing and logging requirements. Events should be forwarded to a syslog server each time an auditable event occurs to ensure event capture. This can be accomplished for Windows, Macintosh, Unix, and many Linux variants [20].

Use the logging capability of the firewall and consider the use of additional software to analyze firewall logs. There is a free product that can perform this analysis available from Digitalbond 
called Portaledge [21]. It requires the PI Server from OSIsoft, the appropriate OSIsoft license, and reads logs from Cisco PIX and Juniper firewalls.

Add IDS/IPS capability for each security zone with coverage that monitors traffic crossing the firewalls to detect potential intrusions attempting to enter the zone. Additional IDS/IPS systems can be used to cover each network segment within a security zone to detect insider intrusion attempts.

The IDS for the ICS/DCS/SCADA network should be capable of monitoring control system protocols in use at the site such as Modbus TCP, DNP3, ODVA, Ethernet/IP ICCP, and others.

SNORT is a popular open source IDS system with signatures available for DNP3, Ethernet/IP, ModBus TCP, and some general SCADA vulnerabilities from Digital Bond[11] The general vulnerability signature set identifies attacks on previously disclosed control system vulnerabilities. There is no charge for the download, but registration as a digitalbond.com subscriber is required. Some commercial vendors now support the DigitalBond Quickdraw rules in their IDS offerings including: 3com/Tipping Point, Cisco, Counterpane/BT, Fortinet, Industrial Defender, ISS/IBM, Juniper, McAfee, Secureworks, Symantec, and Tenable Security.

Industrial Defender also offers a SNORT-based Network Intrusion Detection System (NIDS) that is aware of Modbus TCP, DNP3, ODVA, and Ethernet/IP ICCP[12]. The SNORT sensor device is designed to report to Industrial Defender's centralized Security Event Monitor (SEM).

Limit the use of IPS and rely on IDS and/or log analysis for detection and the determination of appropriate actions to take. While IPS has automatic response capabilities, they may interfere with ICS/SCADA operations creating more problems than they are stopping and should be considered potentially dangerous unless clearly proven safe.

\section{Issues/Clarification}

A few custom systems have emerged from research that are ICS/DCS/SCADA protocol aware, but are limited in the protocols supported. A survey of SCADA-specific IDS systems is available [10].

IDS/IPS capabilities must be fully tested before deployment to ensure they do not interfere with ICS/SCADA systems.

\section{COMMUNICATIONS LINKS}

\section{Definition}


A communications link, or channel, is a single path provided by a transmission medium used to transport analog or digital information between a source and receiver.

Commonly used transmission paths used to support communications include guided media such as copper wire and fiber optics and unguided media such as radio, microwave, and lasers. Services commonly used to support ICS/SCADA communications include: 1) Dial-up telephone lines; 2) Leased/Owned dedicated lines; 3) Microwave; 4) Satellite; 5) Radio; 6) Wireless networks; and 7) The Internet.

While each media type and/or service is susceptible to various security problems (e.g., eavesdropping, interception, re-direction, re-play, and modification), the major concern should be focused on determination of the requirements that are important to the organization: Consideration must be given to: 1 ) secrecy, or confidentiality of the transmission; 2) authentication of the sender and/or receiver; 3 ) proof that the message received was originated by the sender (i.e., non-repudiation) and; 4) that the message has not been altered in transit (integrity control).

Encryption is used to provide secrecy/confidentiality. Strong authentication (at least two-factor) is used to establish identity. Non-repudiation can be provided by a cryptographic digital signature, and integrity is provided by a hash function to create a message digest or Message Authenticating Code (MAC).

\section{Recommendations}

Cryptography should be used to provide one, or more, of the required protections. However, it is important to use proven cryptographic algorithms that are well known, are not themselves secret, and have stood the test of time. Examples include NIST approved algorithms such as the Advanced Encryption Standard (AES), Triple-Data Encryption Algorithm (TDEA), or Triple Data Encryption Standard (TDES) for secrecy, CMAC for authenticity, and Secure Hash Standard (SHS256,384 , or 512 ) for integrity. A more detailed list of algorithms recommended by NIST for Smart Grid Cyber Security is available [23].

\section{ENCRYPTION}

\section{Definition}

Encryption is the process of changing plaintext into ciphertext through the use of a cryptographic algorithm [7].

There are two major types of encryption, symmetric key and asymmetric key. The first, symmetric key, uses one shared key to encrypt and the same key to decrypt. The second, asymmetric key, uses different keys for encryption and decryption. It is also known as public key encryption since one key is publicly known and the other is kept secret. The strength of the 
encryption, or its resistance to breakage depends on the key length, the quality of the algorithm and keeping the key secret (in symmetric encryption) or keeping the private key secret (in asymmetric encryption).

The infrastructure to implement public-key encryption includes software and hardware, of course, as well as a set of policies and procedures to be followed. The systems involved may include a component (called a Certificate Authority) that manages digital certificates, and another (called a Registration Authority) that uses this information in routine operation. The infrastructure (called a PKI) is not yet standardized, and is not yet widely implemented in the utility environment.

Encryption can be a double edged sword. While it can perform necessary and useful service for the system being protected, attackers are more frequently using encryption to hide information from (and therefore to bypass) firewalls and/or network IDS systems. Therefore, sites should consider strategies that can identify legitimate and rogue encryption so traffic containing rogue encryption can be blocked. Another option is to disallow encrypted files through the firewall. One way this can be accomplished is by using in-line encryptors.

\section{Recommendations}

\section{SECRECY}

Use a strong algorithm (e.g., the Advanced Encryption Standard (AES)) to encrypt traffic that travels over communications links that are not under the direct control of your organization. That is, encrypt link traffic that passes through facilities/over media owned by others or where the media is not physically secured. This should be a default requirement for any wireless communications.

\section{INTEGRITY}

Integrity is "guarding against improper information modification or destruction, and includes ensuring information non-repudiation and authenticity." [7]

At a minimum one should implement controls to ensure data integrity on important processes and critical path, e.g., the connection between the control room and field devices. NIST approved algorithms for generating secure hash-based message authentication codes (HMAC) (e.g. SHA-1, SHA-224, SHA-256, SHA-384, and SHA-512) should be used where technically feasible. On low-bandwidth serial connections other protocols may be necessary (e.g. Secure SCADA Communications Protocol (SSCP) or Streaming Encryption Protocol (SEP)). 


\section{ACCESS CONTROL AND AUTHENTICATION}

\section{Definition}

Access control is the process of granting or denying specific requests to: 1) obtain and use information and related information processing services; and 2) enter specific physical facilities (e.g., federal buildings, military establishments, border crossing entrances, etc.).

Authentication is the process of verifying the identity or other attributes claimed by or assumed of an entity (user, process, or device), or to verify the source and integrity of data [9].

\section{Recommendations}

Implement access control at all entry points to the enterprise/control center/ICS/SCADA networks. Authenticate all users attempting access to a protected security zone using a strong authentication mechanism (e.g., Virtual Private networking (VPN), two-factor authentication). Avoid the use of insecure applications such as plaintext telnet and ftp replacing them with secure versions that perform the same functions. If a device cannot support secure login, restrict IP addresses that can access these devices.

\section{TOOLS THAT SUPPORT THE RECOMMENDATIONS}

There are a number of tools that can be used to implement the proposed recommendations. Some tool sets are robust while others are much more limited. The SANS Institute has developed a list of tools that have been confirmed by users to automate part or all of the SANS 20 Critical Security Controls [21]. 


\section{REFERENCES}

[1] Kissel, R., editor, "Glossary of Key Information Security Terms", NISTIR Report No. 7298, Revision 1, National Institute of Standards and Technology, February 2011, pg 68. Available at: http://csrc.nist.gov/publications/PubsNISTIRs.html

[2] Zerbst, J-T., E. Hjelmvic, and I. Rinta-Jouppi, "Zoning Principles in Electricity Distribution and Energy Production Environments", Proceedings of the $20^{\text {th }}$ International Conference on Electricity Distribution, Prague, June 8-11, 2009. Available at:

http://www.cired.be/CIRD09/pdfs/CIRED2009 0838 Paper.pdf

[3] ANSI/ISA 99.02.01-2009, "Security for Industrial Automation and Control Systems", American National Standards Institute, 2009. Available for a fee at:

http://www.isa.org/Template.cfm?Section=Standards2\&template=/Ecommerce/ProductDisplay .cfm\&ProductID $=10234$

[4] Kissel, R., editor, "Glossary of Key Information Security Terms", NISTIR Report No. 7298, Revision 1, National Institute of Standards and Technology, February 2011.pg 61. . Available at: http://csrc.nist.gov/publications/PubsNISTIRs.html

[5] Kissel, R., editor, "Glossary of Key Information Security Terms", NISTIR Report No. 7298, Revision 1, National Institute of Standards and Technology, February 2011, pg. 77. . Available at: http://csrc.nist.gov/publications/PubsNISTIRs.html

[6]Kissel, R., editor, "Glossary of Key Information Security Terms", NISTIR Report No. 7298, Revision 1, National Institute of Standards and Technology, February 2011, pg. 102. . Available at: $\underline{\text { http://csrc.nist.gov/publications/PubsNISTIRs.html }}$

[7] Kissel, R., editor, "Glossary of Key Information Security Terms", NISTIR Report No. 7298, Revision 1, National Institute of Standards and Technology, February 2011, pg. 68. . Available at: http://csrc.nist.gov/publications/PubsNISTIRs.html

[8] Kissel, R., editor, "Glossary of Key Information Security Terms", NISTIR Report No. 7298, Revision 1, National Institute of Standards and Technology, February 2011, pg. 4. . Available at: http://csrc.nist.gov/publications/PubsNISTIRs.html

[9] Kissel, R., editor, "Glossary of Key Information Security Terms", NISTIR Report No. 7298, Revision 1, National Institute of Standards and Technology, February 2011, pg. 14. . Available at: http://csrc.nist.gov/publications/PubsNISTIRs.html

[10] Zhu, B., and S. Sastry, "SCADA-Specific Intrusion Detection/Prevention Systems: A Survey and Taxonomy", First Workshop on Secure Control Systems, part of $13^{\text {th }}$ International 
Conference on Hybrid Systems; Computation and Control. April 12, 2010, Stockholm, Sweden. Available at: https://www.truststc.org/conferences/10/CPSWeek/program.htm

[11] Digital Bond Quickdraw SCADA IDS Signatures. Available at:

http://www.digitalbond.com/tools/quickdraw/

[12] Industrial Defender Network Intrusion Detection System. Available at:

http://www.industrialdefender.com/products/nids.php

[13] Kurt Dillard, "Intrusion Detection FAQ: What is a Bastion Host". SANS Institute. Available at: http://www.sans.org/security-resources/idfaq/bastion.php

[14] Gite, V., "Configure Linux As Bastion Host", NnixCraft web site, June 26, 2009. Available at: http://www.cyberciti.biz/faq/linux-bastion-host/

[15] Titania Nipper, Network switch, router, and firewall auditing software. Available at: https://www.titania-security.com/nipper/overview

[16] OSSEC Documentation Page. Available at: http://www.ossec.net/doc/

[17] Robertson, Chad, "Practical OSSEC", SANS InfoSec Reading Room, July 5, 2011. Available at: http://www.sans.org/reading room/top25.php

[18] Tripwire home page. Available at: http://www.tripwire.com/

[19] "U.S. Code-Cracking Agency Works as if Compromised", Reuters Canada, December 16, 2010. Available at: http://ca.reuters.com/article/technologyNews/idCATRE6BF6BZ20101216

[12] Nawyn, Kenneth, "A Security Analysis of System Event Logging with Syslog", SANS Reading Room, October 31, 2003. Available at:

http://www.sans.org/reading room/whitepapers/logging/

[21] Protaledge is available at: http://www.digitalbond.com/tools/portaledge

[22] A list of the 17 user vetted tools is available at: http://www.sans.org/critical-securitycontrols/user-tools.php

[23] National Institute of Standards and Technology, "Guidelines for Smart Grid Cyber Security: Vol. 1, Smart grid Cyber Security Strategy, Architecture, and High-Level Requirements", NISTIR 7628, August 2010. Available at: http://csrc.nist.gov/publications/nistir/ir7628/mistir-

7628 vol1.pdf

[A1] Hadley, M.D., J.B. McBride, T.W. Edgar, L.R. O’Neil, and J.D. Johnson, “Securing Wide Area Measurement Systems", PNNL-16705, Pacific Northwest National Laboratory, June 2007.

Available at: http://energy.gov/oe/downloads/securing-wide-area-measurement-systems 
[A2] ASAP-SG Wide-Area Monitoring, Protection, and Control (Synchrophasor) Security Profile (Draft) v0.08 is available at:

http://www.smartgridipedia.org/images/5/5e/WAMPAC Security Profile - v0 08.pdf

[A3] ASAP-SG Third Party Data Access Security Profile v1.0 is available at:

http://www.smartgridipedia.org/images/6/65/3PDA Security Profile - v1 0 - 20110518.pdf

[A4] ASAP-SG AMI Security Profile v2.0 is available at:

http://www.smartgridipedia.org/images/9/90/AMI_Security_Profile__-v2_0.pdf 


\section{ICS/SCADA SPECIFIC BIBLIOGRAPHY}

The following documents provide guidance specific to ICS/DCS/SCADA cyber security protection.

British Columbia Institute of Technology (BCIT), "Good Practice Guide on Firewall Deployment for SCADA and Process Control Networks (Prepared for National Infrastructure Security Coordination Centre)", National Infrastructure Security Co-ordination Centre (NISCC), February 2005. Available at: http://www.cpni.gov.uk/docs/re-20050223-00157.pdf

A widely referenced guide covering multiple types of firewalls, descriptions of 8 alternatives for segregating the process control/SCADA network for an enterprise network, recommended firewall rules for specific services, and addresses firewall issues with regard to data historians, remote access requirements, and multicast traffic.

Byers, E., B. Chauvin, J. Karsh, D. Hoffman, and N. Kube, "The Special Needs of SCADA/PCN Firewalls: Architecture and Test Results", $10^{\text {th }}$ IEEE Conference on Emerging Technologies and Factory Automation, Catania, Italy, 2005. pp. 884-892. Available at:

http://ieeexplore.ieee.org/xpls/abs all.jsp?arnumber=1612765 and/or from:

http://www.tofinosecurity.com/professional/special-needs-scadapcn-firewalls-architecturesand-test-results (free registration required).

Argues that current SCADA/PCN firewall are often poorly implemented, but that they can be successfully implemented based on test results for the configuration of four firewalls.

Homeland Security, "Recommended Practice for Patch Management of Control Systems", Department of Homeland Security, December 2008. Available at: http://www.uscert.gov/control systems/practices/Recommended Practices.html

Focused on the overall patch management issue for ICS/SCADA systems including the requirements for a successful patch management program, required analysis prior to patching, and patch deployment processes.

National Security Agency, "A Framework for Assessing and Improving the Security Posture of Industrial Control Systems (ICS)", Version 1.1, Systems and network Analysis Center, National Security Agency, August 20, 2010. Available at:

http://www.nsa.gov/ia/guidance/security configuration guides/ics.shtml

Covers the mapping of all ICS networked assets and communications links, assessment of the consequences of a loss of assets, an assessment of threat sources attack vectors and attack difficulty, and prioritizing defensive measures. 
Stouffer, K., J. Falco, and K. Scarfone, "Guide to Industrial Control Systems Security", National Institute of Standards and Technology, Special Publication 800-82, dated June 2011. Available at: http://csrc.nist.gov/publications/PubsSPs.html

SP 800-82 covers an overview of industrial control systems, describes threats and vulnerabilities, how to create a business case for implementing ICS protection, covers the elements for establishing an ICS security program, developing a firewall-protected network architecture, and recommends a list of security controls that are recommended for implementation.

Peterson, D., "Intrusion Detection and Cyber Security Monitoring of SCADA and DCS Networks", ISA, 2004. Available at: http://www.techrepublic.com/whitepapers/intrusion-detection-andcyber-security-monitoring-of-scada-and-dcs-networks/126355

Now dated, covers existing use of IDS and log monitoring in SCADA systems, limitations due to lack of applications and protocol awareness, and suggests the development of SCADA aware IDS signature (which is beginning to occur). Urges the analysis of existing SCADA logs.

Department of Homeland Security CPNI "Cyber Security Assessments of Industrial Control Systems" November 2010. Available at: http://www.uscert.gov/control systems/pdf/Cyber Security Assessments of Industrial Control Systems.pdf

Describes what elements should be included in a security assessment of industrial control systems. It is descriptive but not prescriptive. This documents aims "to assist asset owners to maximize the return on their investment when commissioning assessments of their ICSs." 


\section{GENERAL BIBLIOGRAPHY}

Cyber Security Operations Centre, "Top 35 Mitigation Strategies", Australian Government Department of Defense, July 21, 2011. Available at:

http://www.dsd.gov.au/infosec/top35mitigationstrategies.htm

National Institute of Standards and Technology, "Recommended Security Controls For Federal Information Systems and Organizations", NIST Special Publication 800-53, Revision 3, August 2009. Available at: : http://csrc.nist.gov/publications/PubsSPs.html

Appendix I of SP 800-53 provide supplemental guidance specifically tailored to the requirements of industrial control systems. 


\section{APPENDIX A - Use Cases}

\section{Use Case 1: Enterprise Network to Operations Network.}

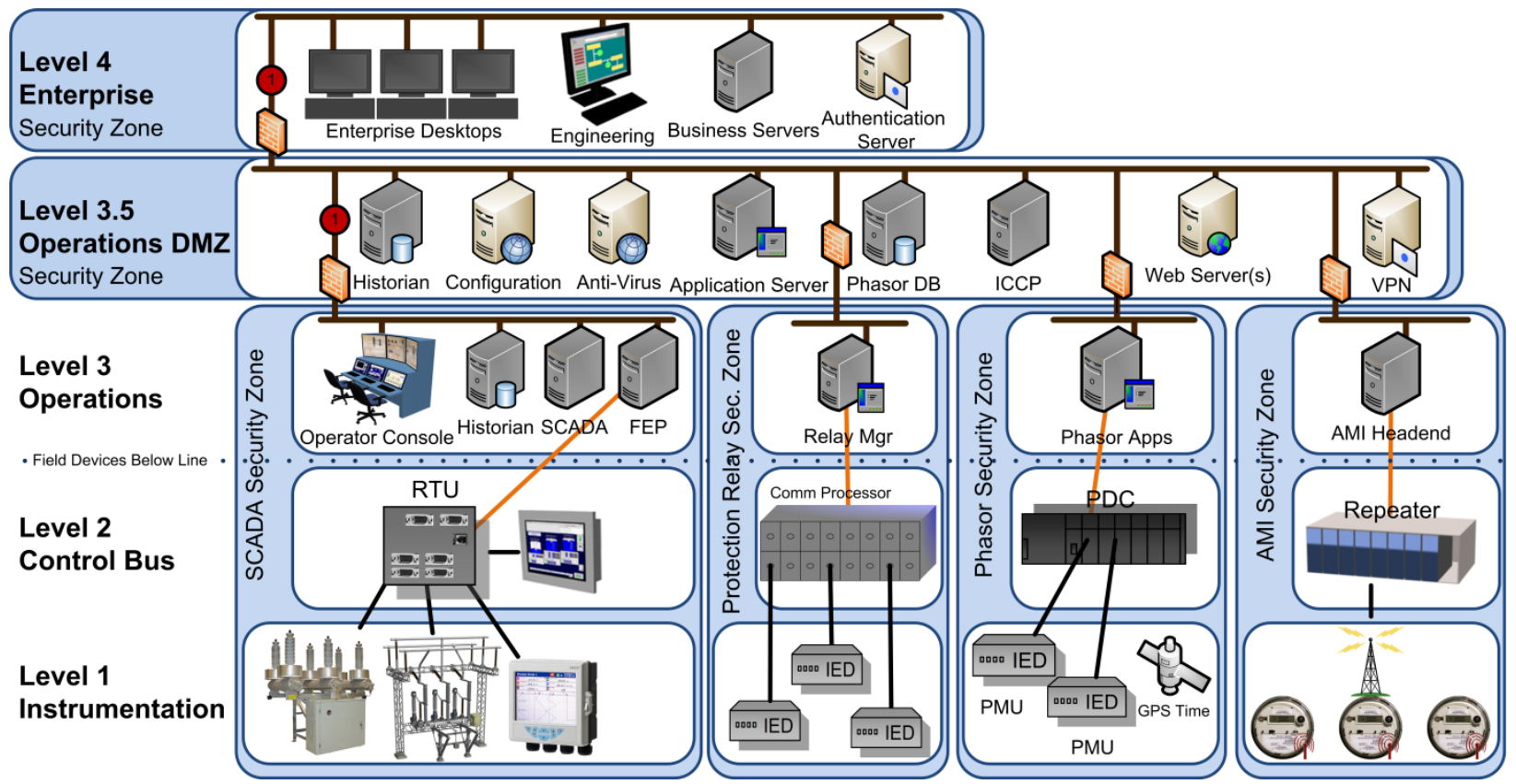

The Enterprise network should be considered a hostile network. It is complex, supports many vulnerable protocols and even if strongly protected is susceptible to zero-day attacks. Consequently, the operations network needs to be protected from systems in the enterprise network that may have been compromised. This means that information shared between the enterprise network and the control center (e.g., Historian, patch management servers, etc.) need to be staged in a DMZ with no transit traffic allowed. Where direct access is required, such as from engineering workstations, it should be via strong authentication, such a VPN or using two-factor authentication, accessing workstations should be whitelist allowed, and the number of protocols allowed between the networks should be limited to those few that are critically necessary for operations. Implement IDS within both networks. 
Use Case 2: Operations Network to External Control Center Network (Phasor Data).

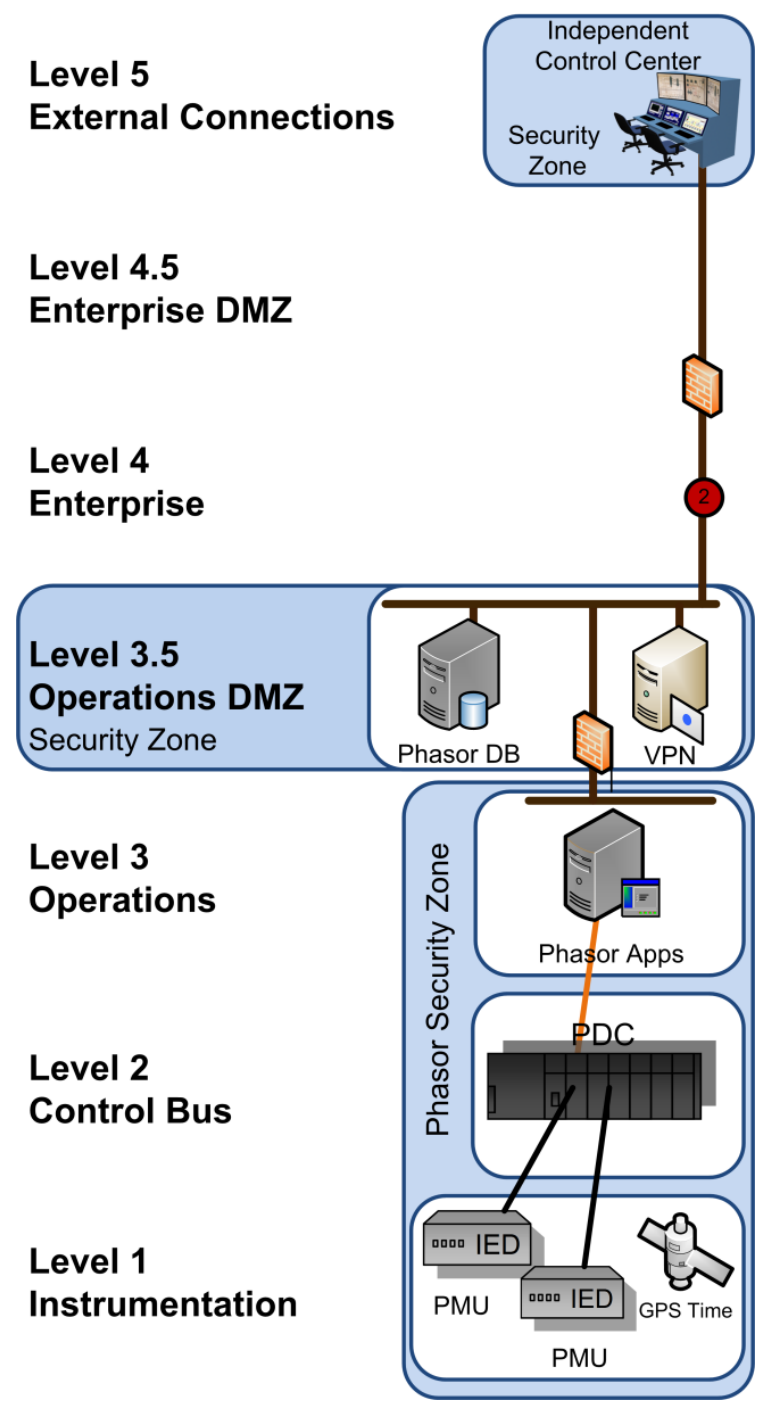

Wide Area measurement Systems (WAMS) security has been addressed in a separate report and will not be repeated here [A1]. This report focuses on risks and vulnerabilities in cyber security, reliability, data quality and human performance as they relate to WAMS. The Advanced Security Acceleration Project for the Smart Grid (ASAP-SG) is developing a security profile titled Wide-Area Monitoring, Protection and Control (Synchrophasor) Security Profile [A2] to further address this use case. It is currently in early draft form. 
Use Case 3: Operations Network to External Control Center Network - ICCP Data.

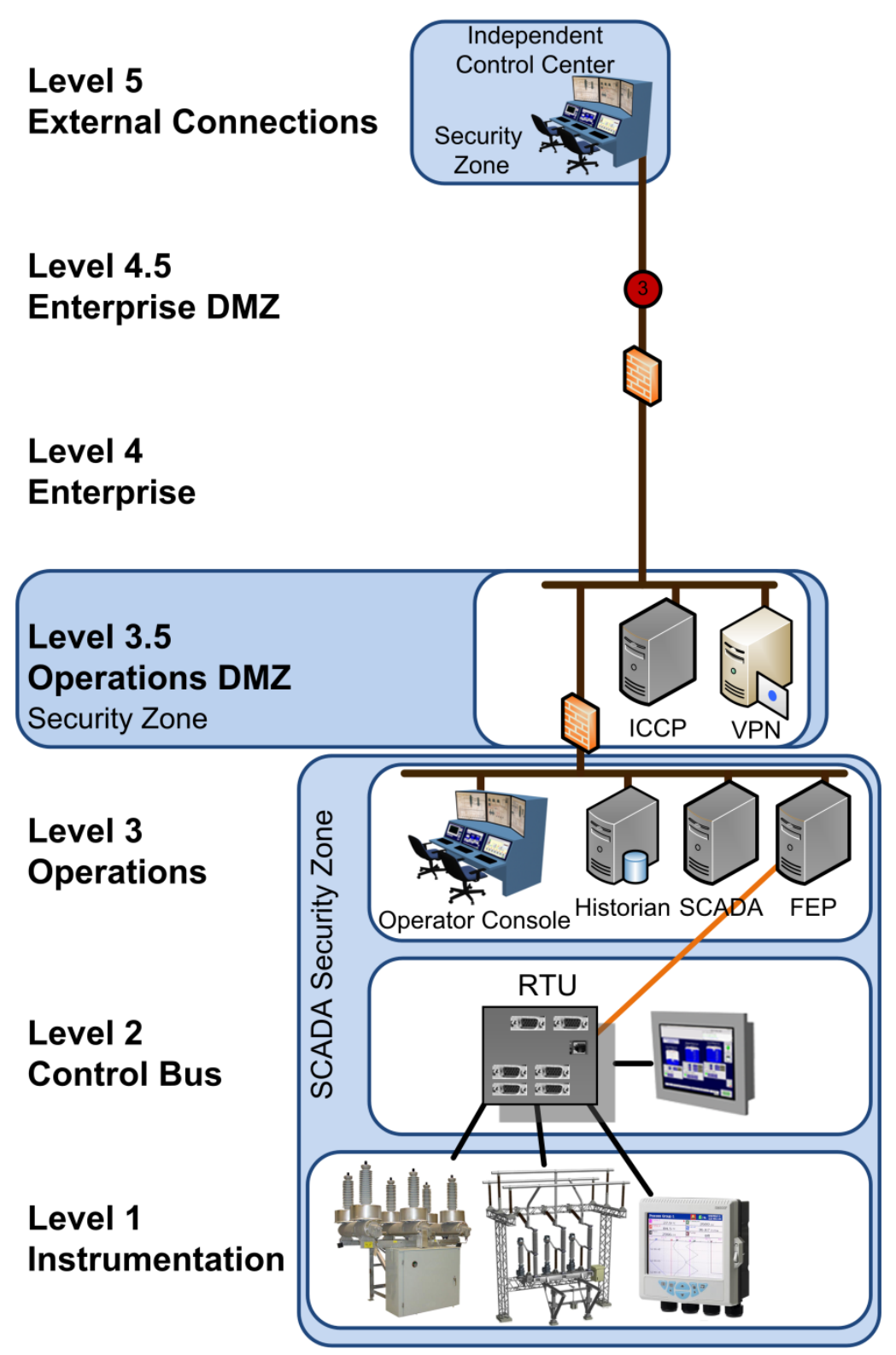

The Inter-Control Center Communications Protocol is used for communications control centers over Wide Area Networks and is subject to the same attacks experienced by other routable protocols. While there is a security enhanced version of ICCP, TASE. 2 that strengthens security, it requires a Public Key Infrastructure, which is not widely implemented in the utility industry. The ASAP-SG Third Party Data Access Security Profile v1.0 [A3] provides detailed information about securing this use case. 
Use case 4: Operations Network to NERC, Regulatory Data.

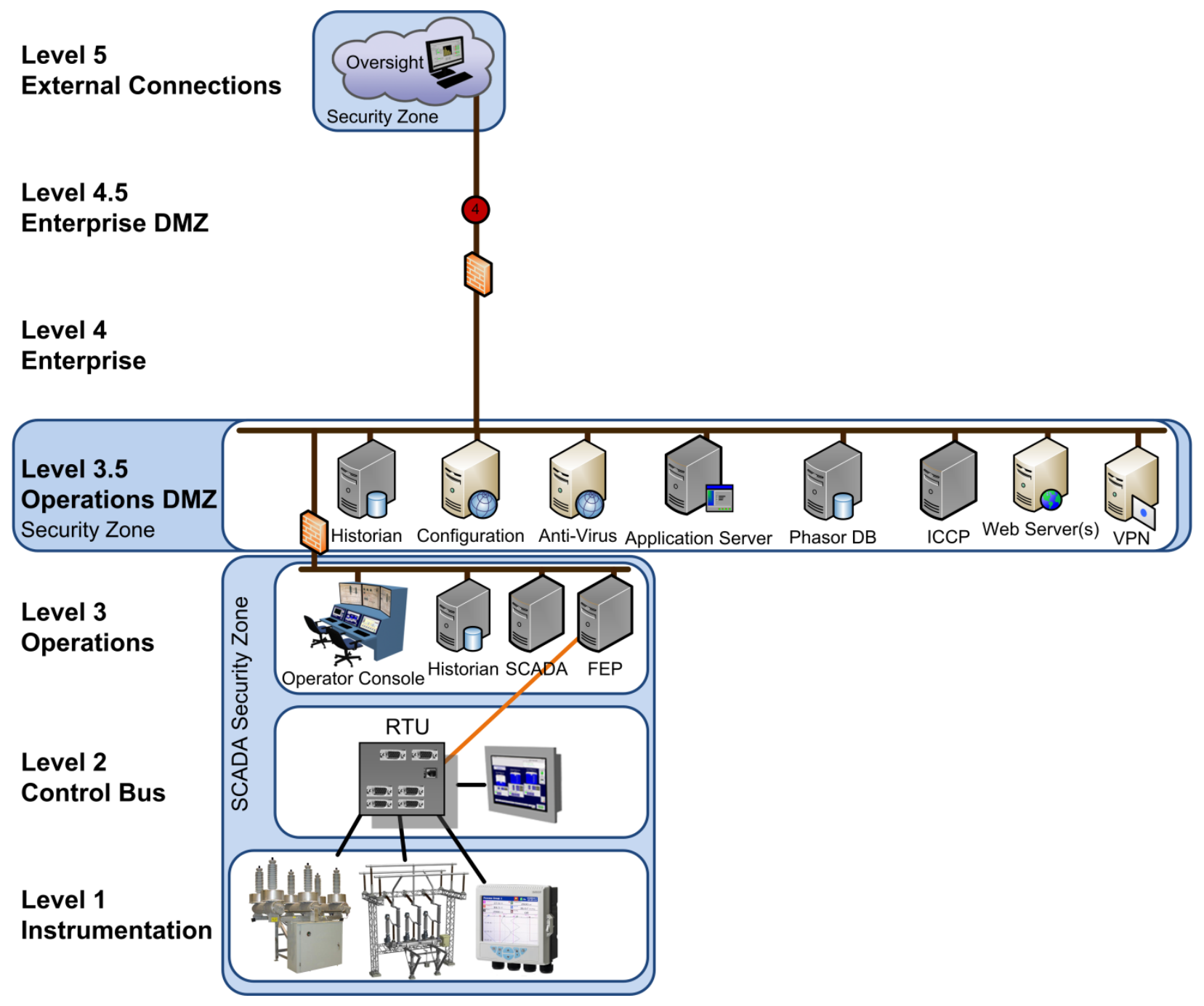

Sites are required to report to various regulatory bodies (e.g., NERC). Reporting is typically electronic via a communications link that poses a potential threat to the site. The threat can be minimized by isolating the system that performs the reporting, typically a historian such that required reports are staged to the reporting server and no transit traffic is allowed from the server to the control or ICS/SCADA networks. Reports should be generated, deposited on the server and then either pushed to the regulator or made accessible to the regulator. If secrecy, integrity, and/or non-repudiation is required, it should be implemented with robust cryptography.

The ASAP-SG Third Party Data Access Security Profile v1.0 [A3] provides detailed information about securing this use case. 


\section{Use Case 5: Operations Network to Vendor Network, Support \& Maintenance.}

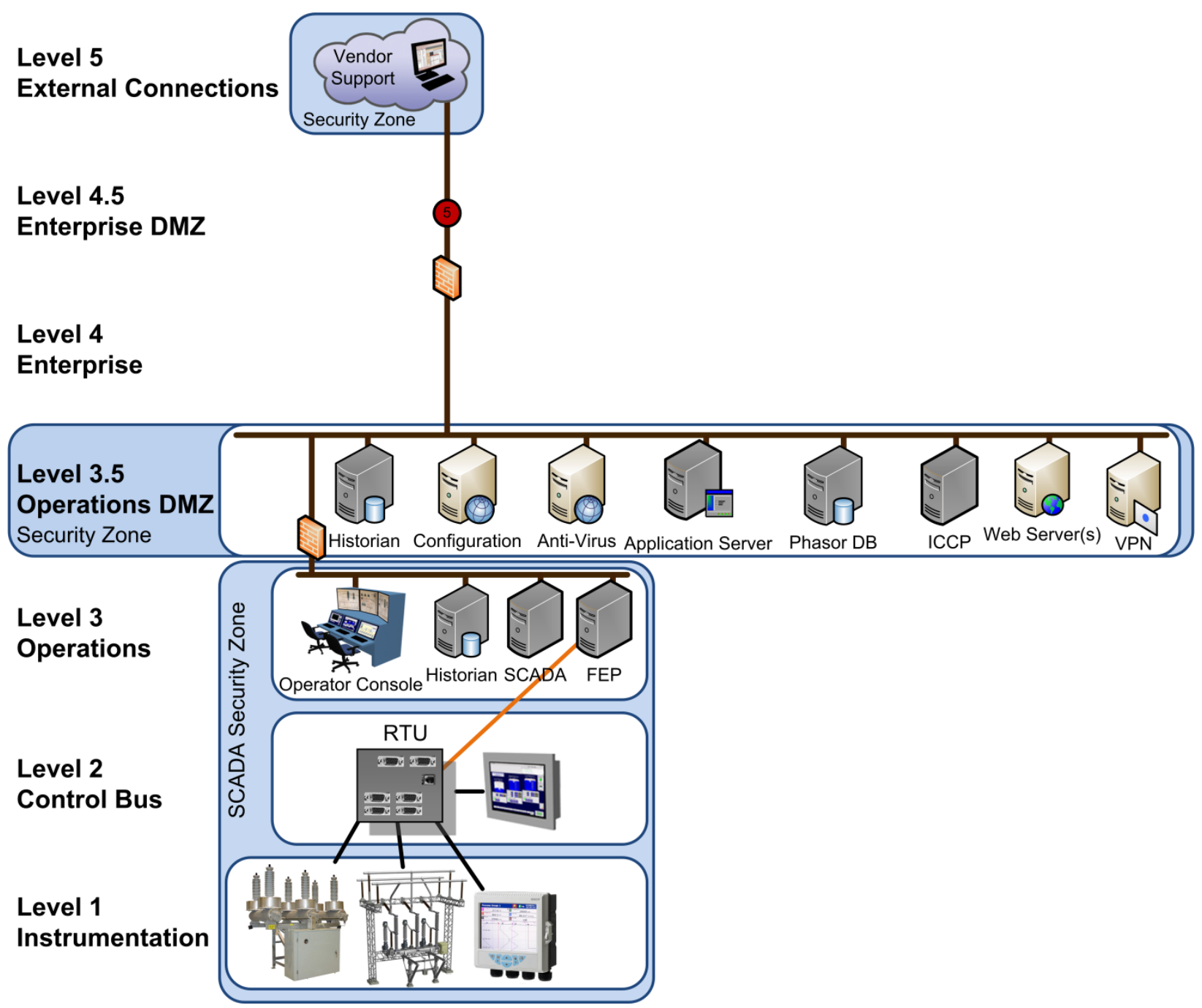

Third party access to cyber resources within the control center and/or remote sites is typically required to support installations, upgrades, troubleshooting, and remedial maintenance. The primary issue with third party access is with remote communication links established to support vendor activities. Such a link provides a potential path for the introduction of malware into the system being serviced. Remote access should be via VPN and/or use of strong authentication. Wherever possible, the accessed system should be isolated from other systems on the network during the work activity, any vendor performing work onsite should be escorted, access should be at the lowest privilege level feasible and all work activities should be observed by technical staff.

The ASAP-SG Third Party Data Access Security Profile v1.0 [A3] provides detailed information about securing this use case. 
Use Case 6: Operations Network to Advanced Metering Infrastructure

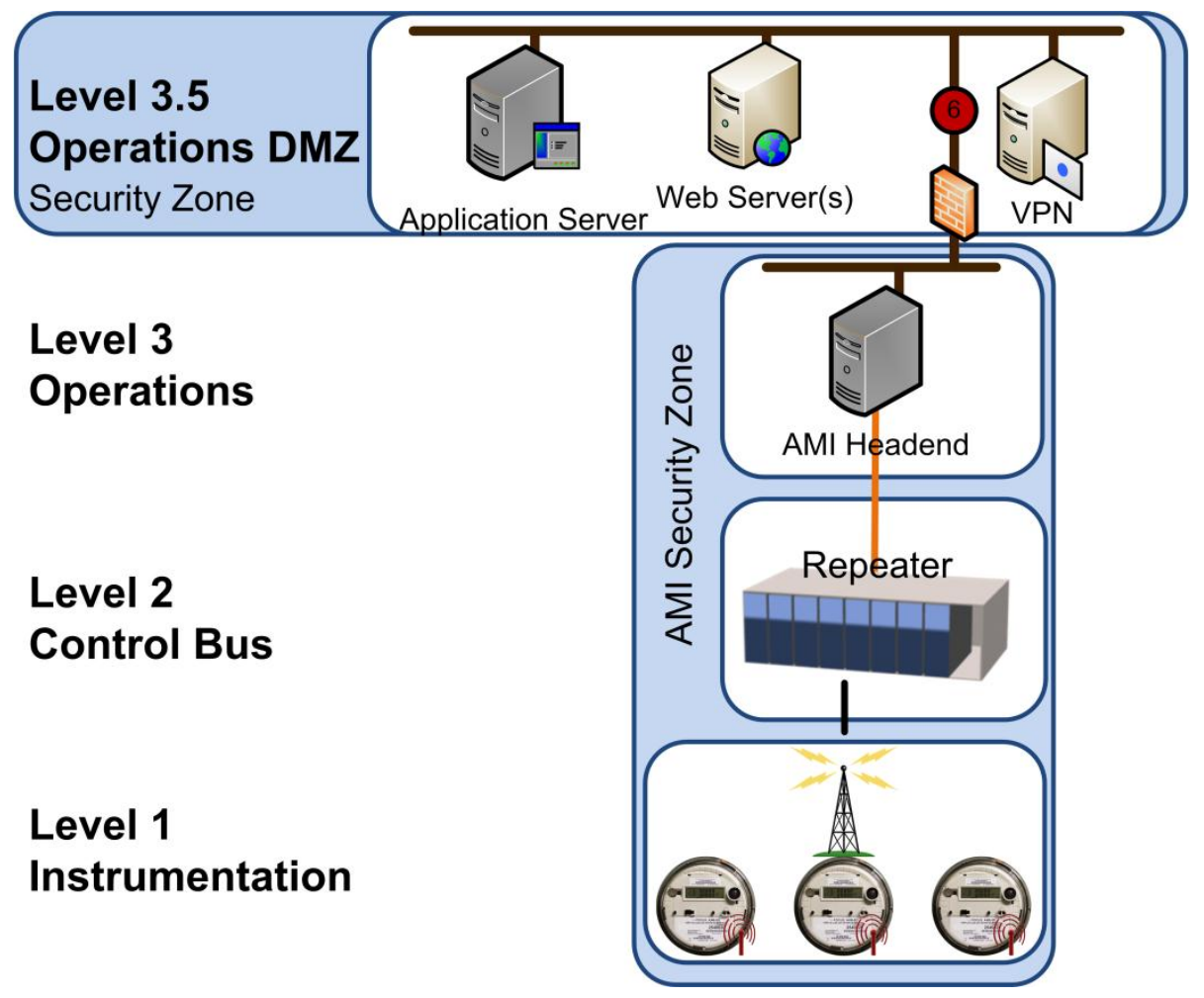

Automated meter reading using wireless technology is rapidly growing and with the introduction of "Smart grid" technology allows fine-grained control over consumer loads. However, the introduction of wireless technology, whether in the smart grid or as part of the enterprise, control center, or remote site infrastructure bring with it serious security concerns. The wireless network should be isolated from other networks by a firewall and implemented with strong encryption and authentication. Many AMI vendors use licensed frequencies and proprietary protocols to gather meter data. The adherence to security of these solutions can vary dramatically and special consideration should be given when evaluating these systems. The ASAP-SG AMI Security Profile v2.0 [A4] provides detailed information about securing this use case. 


\section{APPENDIX B: Crosswalk with NISTIR 7628}

\section{Background}

The NISTIR 7628 is a hefty and detailed document that provides high-level guidance on securing smart grid systems. It can be used to find guidance on all aspects of cyber security for smart grid systems. The Secure Data Transfer Guidance for Industrial Control and SCADA Systems is meant to be a helpful hands-on guide to securely moving data around a network. It is not intended to be as broadly encompassing of security issues as the NISTIR 7628. Consequently, not all of the requirements recommended in the NISTIR 7628 are found in the recommendations of this document.

\section{Crosswalk}

This appendix is intended to highlight how the recommendations given in this document relate to the guidance given in the NISTIR 7628 .

\section{Secure Data Transfer}

- Generic SCADA Architecture

\begin{tabular}{|c|}
\hline $\begin{array}{l}\text { Segregation } \\
\text { - Disallow Unneeded Protocols }\end{array}$ \\
\hline Demilitarized Zone (DMZ) \\
\hline
\end{tabular}

Firewalls

\section{NISTIR 7628}

- Vulnerability C.4.1.1 Inadequate Security Architecture and Design Security Zones

- Vulnerability C.4.3.5 Inadequate Network

- Vulnerability C.4.3.2 Unneeded Services Running

- Vulnerability C4.3.6 Inappropriate Protocol Selection

- DHS - 2.15.28 External Access Protection

- DHS - 2.8.7 Boundary Protection

- DHS - 2.8.7.2 Supplementary Guidance

- DHS - 2.15.15 Information Flow Control

- DHS - 2.15.28 External Access Protections

- Vulnerability C.4.3.5 Inadequate Network Segregation

\begin{tabular}{|c|c|}
\hline Protect all entry points & - DHS - 2.8.18 System Connections \\
\hline $\begin{array}{l}\text { Intrusion Detection } \\
\text { - Logs } \\
\text { - Intrusion Detection Systems }\end{array}$ & $\begin{array}{l}\text { - DHS - 2.14.3 Malicious Code Protection } \\
\text { - DHS - 2.14.4 System Monitoring Tools and } \\
\text { Techniques } \\
\text { - Vulnerability C } 43.3 \text { Insufficient Log } \\
\text { Management } \\
\text { - Vulnerability C.4.3.4 Inadequate Anomaly } \\
\text { - Tracking } \\
\text { - Vulnerability C.4.3.9 Physical Access to Device }\end{array}$ \\
\hline Communication Links & - DHS - 2.8.8 Communications Integrity \\
\hline Cryptographic Secrecy & $\begin{array}{l}\text { - } \text { DHS - 2.8.9 Communications Confidentiality } \\
\text { - } \text { DHS - 2.8.7 Boundary Protection }\end{array}$ \\
\hline
\end{tabular}




\begin{tabular}{|c|c|}
\hline & - DHS - 2.15.24 Remote Access \\
\hline Cryptographic Integrity & $\begin{array}{l}\text { - } \text { DHS - 2.8.7 Boundary Protection } \\
\text { - } \text { DHS - 2.14 System and Information Integrity } \\
\text { - } \text { DHS - 2.14.7 Software and information Integrity } \\
\text { - } \text { DHS - 2.15. Remote Access } \\
\text { - DHS - 2.15.28 External Access Protections } \\
\text { - Vulnerability C.5.1 Inadequate Integrity } \\
\text { Checking }\end{array}$ \\
\hline Cryptographic Authenticity & - DHS - 2.8.20 Message Authenticity \\
\hline Use Validated Cryptography & - DHS - 2.8.12 Validated Cryptography \\
\hline Access Control and Authentication & $\begin{array}{l}\text { - } \text { DHS - 2.15 Access Control } \\
\text { - } \text { DHS - 2.15.28 Remote Access }\end{array}$ \\
\hline
\end{tabular}




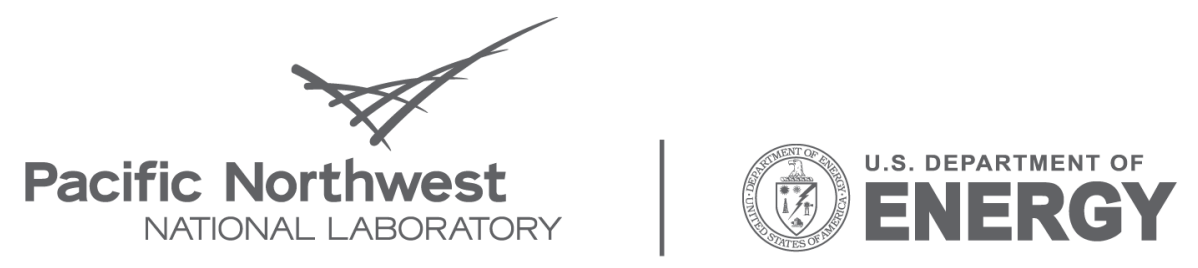

Proudly Operated by Battelle Since 1965

902 Battelle Boulevard

P.O. Box 999

Richland, WA 99352

1-888-375-PNNL (7665)

www.pnl.gov 